\title{
THREE-DIMENSIONAL MHD SIMULATION OF THE 2003 OCTOBER 28 CORONAL MASS EJECTION: COMPARISON WITH LASCO CORONAGRAPH OBSERVATIONS
}

\author{
Ward B. Manchester IV, ${ }^{1}$ Angelos Vourlidas, ${ }^{2}$ Gábor Tóth, ${ }^{1}$ Noé Lugaz, ${ }^{3}$ Ilia I. Roussev, ${ }^{3}$ Igor V. Sokolov, ${ }^{1}$ \\ Tamas I. Gombosi, ${ }^{1}$ Darren L. De Zeeuw, ${ }^{1}$ and Merav Opher ${ }^{4}$ \\ Received 2007 December 19; accepted 2008 May 19
}

\begin{abstract}
We numerically model the coronal mass ejection (CME) event of 2003 October 28 that erupted from AR 10486 and propagated to Earth in less than $20 \mathrm{hr}$, causing severe geomagnetic storms. The magnetohydrodynamic (MHD) model is formulated by first arriving at a steady state corona and solar wind employing synoptic magnetograms. We initiate two CMEs from the same active region, one approximately a day earlier that preconditions the solar wind for the much faster CME on the 28th. This second CME travels through the corona at a rate of over $2500 \mathrm{~km} \mathrm{~s}^{-1}$, driving a strong forward shock. We clearly identify this shock in an image produced by the Large Angle Spectrometric Coronagraph (LASCO) $\mathrm{C} 3$ and reproduce the shock and its appearance in synthetic white-light images from the simulation. We find excellent agreement with both the general morphology and the quantitative brightness of the model CME with LASCO observations. These results demonstrate that the CME shape is largely determined by its interaction with the ambient solar wind and may not be sensitive to the initiation process. We then show how the CME would appear as observed by wide-angle coronagraphs on board the Solar Terrestrial Relations Observatory (STEREO) spacecraft. We find complex time evolution of the white-light images as a result of the way in which the density structures pass through the Thomson sphere. The simulation is performed with the Space Weather Modeling Framework (SWMF).
\end{abstract}

Subject headings: MHD — shock waves — Sun: coronal mass ejections (CMEs)

Online material: mpeg animation

\section{INTRODUCTION}

The October-November period of 2003 saw some of the most energetic solar flares and coronal mass ejections of any solar cycle. These eruptive events came to be known as the Halloween Events and included 11 X-class flares, six radiation storms, and four geomagnetic storms. These eruptions originated from three active regions, ARs 10484, 10486, and 10488, of which AR 10486 was the largest, most complex, and most active, producing 12 major coronal mass ejections (CMEs). Three of these CMEs that occurred on October 28, 29, and November 4 were particularly energetic with speeds in excess of $2000 \mathrm{~km} \mathrm{~s}^{-1}$ and were associated with $\mathrm{X} 17, \mathrm{X} 10$, and X28 flares, respectively. The October $28 \mathrm{CME}$ was very geoeffective and damaged satellites, diverted airplane routes, caused power failures in Sweden, disrupted long-distance radio communications, and caused northern lights (aurorae borealis) as far south as Florida. The November $4 \mathrm{CME}$ was the most energetic of the Halloween Events, but it occurred while AR 10486 was on the western limb so that the CME was less geoeffective as the CME was not directed toward Earth.

Because of their extreme nature, the Halloween Events have been the subject of a great deal of study, both observational as well as theoretical, and modeling efforts. The October $28 \mathrm{CME}$ in particular was well observed from Sun to Earth, beginning with the vector magnetic field of AR 10486 (Liu et al. 2005) and surface flow measurements (Yang et al. 2004; Deng et al. 2006). For this event, flare loops were observed by TRACE (Su et al. 2006), and the global coronal field has been reconstructed from data taken from the Michelson Doppler Imager (MDI) aboard the Solar and Heliospheric Observatory $(\mathrm{SOHO})$ spacecraft (Liu \& Hayashi

\footnotetext{
1 Center for Space Environment Modeling, University of Michigan, Ann Arbor, MI 48109.

2 Naval Researach Laboratory, Washington, DC 20375.

3 Institute For Astronomy, University of Hawaii, Honolulu, HI 96822.

4 George Mason University, Fairfax, VA 22030.
}

2006). Dense plasma associated with the October 28 CME has been observed to propagate past the Earth by the Solar Mass Ejection Imager (SMEI; Jackson et al. 2006) and was also detected by interplanetary scintillation (Tokumaru et al. 2007). Finally, the event was observed in situ by Wind and the Advanced Composition Explorer (ACE; Skoug et al. 2004; Zurbuchen et al. 2004), which reveal magnetohydrodynamic (MHD) quantities in the solar wind as well as ion abundances.

Efforts to simulate the Halloween Events include those of Intriligator et al. (2005), who modeled the propagation of the CME plasma from $2.5 R_{\odot}$ to $10 \mathrm{AU}$ with a three-dimensional (3D) kinematic model. Krall et al. (2006) modeled the 2003 October 28-30 period with a 1.5-dimensional reduced MHD model that describes the self-similar expansion of a magnetic flux rope. In this case, the authors also coupled the heliospheric results with a 3D magnetosphere code and compared it with simulations driven by the observed solar wind data. Liu \& Hayashi (2006) modeled the CME eruption in the solar corona with density and pressure perturbations. Dryer et al. (2004) and Wu et al. (2005) both modeled CME-driven shock propagation and predicted the shock arrival times with reasonable success. This work that models a specific event marks a clear distinction from non-event-specific CME simulations (e.g., Usmanov \& Dryer 1995; Riley et al. 2002; Manchester et al. 2004b; Jacobs et al. 2007) and non-eventspecific Sun-to-thermosphere space weather simulations modeled by the Center for Space Environment Modeling (CSEM; Tóth et al. 2005) and the Center for Integrated Space Weather Modeling (CISM; Luhmann et al. 2004).

More recently, Tóth et al. (2007) modeled the October 28 CME as part of a Sun-to-Earth space weather event that included an indepth description of the resulting geomagnetic storm. This work by Tóth et al. (2007) is groundbreaking in two regards. First, like those by Lugaz et al. (2007) and Cohen et al. (2008) it is the first 3D numerical full MHD simulation to reproduce observed CMEs as they are magnetically driven from active regions in the low 
corona. The earliest attempt to model an observed CME (including the magnetic field) propagating from the Sun to the Earth is that of Wu et al. (1999). This particular simulation is two-dimensional and did not capture the structure of the solar wind or the active region, but did roughly capture the magnetic cloud at 1 AU. More recent modeling efforts have been 3D and capture the structure of the solar wind, but treat CME propagation outside of the magnetosonic point $\left(r \approx 20 R_{\odot}\right.$; Odstrcil et al. 2005). The second noteworthy aspect of the simulation by Tóth et al. (2007) is that it is the first event study to employ a framework to couple several individual codes to model the physical domain extending from the corona to the Earth's upper atmosphere. The framework used was the Space Weather Modeling Framework (SWMF), developed by members of CSEM at the University of Michigan (Tóth et al. 2005). A framework for similar purposes is being developed by CISM at Boston University.

In this paper, we examine the simulation described by Tóth et al. (2007) and concern ourselves with the physical properties of the $\mathrm{CME}$ as it propagates from the low corona to Earth. In particular, the detailed structure of the coronal and solar wind allow us to quantitatively compare the model with Large Angle Spectrometric Coronagraph (LASCO) C2 and C3 observations of the October 28 event. In this case, we validate the accuracy of the simulation in reproducing the speed, mass, appearance (in scattered light), and shock properties of the observed CME. We also make wide-angle, large-elongation, Thomson-Scattered white-light images of the model to show how the CME would appear in the wide angle coronagraphs of the Sun Earth Connection Corona and Heliospheric Imager (SECCHI) instrument on board the STEREO spacecraft. These synthetic images show that the CME appears much different far from the Sun due to scattering affects at large elongation.

The rest of this paper is organized as follows: The SWMF, its components, and numerical techniques are briefly discussed in $\S 2$. Section 3 provides a description of the CME simulation, the results of which are compared to LASCO observations in $\S 4$. In $\S 5$ we show how the CME would appear propagating past the Earth in the SECCHI heliospheric imagers HI1 and HI2. We conclude with a summary and an outlook for future development in $\S 6$.

\section{NUMERICAL METHODS: SWMF AND BATS-R-US}

The SWMF ( Tóth et al. 2005) couples models treating physical domains of the space environment extending from the solar corona to the Earth's upper atmosphere. The model coupling is flexible yet efficient, making faster than real-time space weather simulations feasible on massively parallel computers. Each model has its own dependent variables, a mathematical model, and a numerical scheme with an appropriate grid structure and temporal discretization. The physics domains may overlap with each other, or they can interact through a boundary surface. The SWMF is able to incorporate models from the community and couple them with modest changes in the software of an individual model. The SWMF is a fully functional and documented framework that provides highperformance computational capability to simulate the physics from the low solar corona to the upper atmosphere of the Earth. Currently, the SWMF is composed of nine physics modules and is driven by external data such as magnetograms, flare and CME observations, satellites upstream of the Earth (like ACE, Geotail, and Wind), etc. The SWMF can model any physically meaningful subset of the physics domains and is freely available online. ${ }^{5}$

There were seven SWMF components used in the Halloween storm simulations: Solar Corona (SC), Eruptive Event Generator (EE), Inner Heliosphere (IH), Global Magnetosphere (GM), Inner

\footnotetext{
5 See http://csem.engin.umich.edu/SWMF.
}

Magnetosphere (IM), Ionosphere Electrodynamics (IE), and Upper Atmosphere (UA). For our purposes, the only relevant components are the SC, EE, and IH, which model the corona, solar wind, and CME propagation from the active region to $1 \mathrm{AU}$. Components SC, EE, and IH are based on the BATS-R-US code (Powell et al. 1999; Gombosi et al. 2001), which solves the governing equations of MHD in a conservative finite volume form. Non-ideal MHD terms are included through appropriate source terms. The code uses a limited reconstruction that ensures second-order accuracy away from discontinuities while simultaneously providing the stability that ensures non-oscillatory solutions. In addition, the code employs several approximate Riemann solvers. The resulting scheme solves for the hydrodynamic and electromagnetic effects in a tightly coupled manner that works equally well across several orders of magnitude in plasma $\beta$ (the ratio of plasma pressure to magnetic pressure). The BATS-R-US also uses a relatively simple yet effective block-based adaptive mesh refinement scheme to resolve structures spanning many length scales. The BATS-R-US scales almost linearly to more than 1000 processors for a fixed problem size, and a fully implicit time stepping scheme is incorporated (Tóth et al. 2006) that can be combined with explicit time stepping.

The solar corona domain is a Cartesian box that extends from the surface of the Sun to $-24 R_{\odot}<x, y, z<24 R_{\odot}$, where $R_{\odot}$ is the radius of the Sun. The physics of this domain is described by the equations of MHD, with additional source terms required to take into account the heating and acceleration of the solar wind (Groth et al. 2000; Usmanov et al. 2000). Recently, Cohen et al. (2007) developed a solar wind model based on the empirical relationship between solar wind speed and magnetic flux tube expansion (Wang \& Sheeley 1990), which has been incorporated into the SWMF. Here we use the coronal model presented in Roussev et al. (2003b). At the inner boundary of the SC component, the density, pressure, velocity, and magnetic field are defined at a height just above the transition region. The magnetic field is obtained from a synoptic solar magnetogram. The boundary conditions for the temperature and mass density at the Sun are varied as a function of magnetic field strength to achieve a realistic distribution of fast and slow wind speeds near the Sun and at $1 \mathrm{AU}$. The velocity components at the inner boundary maintain linetying of the magnetic field to the rotating solar surface. Differential rotation is currently neglected. The flow at the outer boundary is usually superfast (faster than the fast magnetosonic speed of the plasma), so no information is propagating inward.

The EE domain is in the solar corona, restricted to the active region responsible for the $\mathrm{CME}$. The $\mathrm{EE}$ in this case takes the form of a nonlinear perturbation of the SC solution, which is made by superposing a modified version of the Titov \& Démoulin (1999) flux rope to AR 10486. The flux rope is in an initial state of force imbalance that drives the eruption. This eruption generator follows from the work of Roussev et al. (2003a), who incorporated the Titov \& Démoulin (1999) flux rope in the BATS-R-US code.

The IH domain extends from its inner boundary at $r=20 R_{\odot}$ to a cube extending to $-240 R_{\odot}<x, y, z<240 R_{\odot}$, which encompasses Earth's orbit. The physics of this domain is described by the equations of ideal MHD, solved in an inertial frame, on a Cartesian grid. The inner boundary conditions of the IH component are obtained from the SC component. The flow at the outer boundary of the IH component is always assumed to be superfast.

\section{HALLOWEEN STORM SIMULATION}

\subsection{Initial State}

Here we summarize the basic description of the CME simulation that was first presented in Tóth et al. (2007). For more details, 

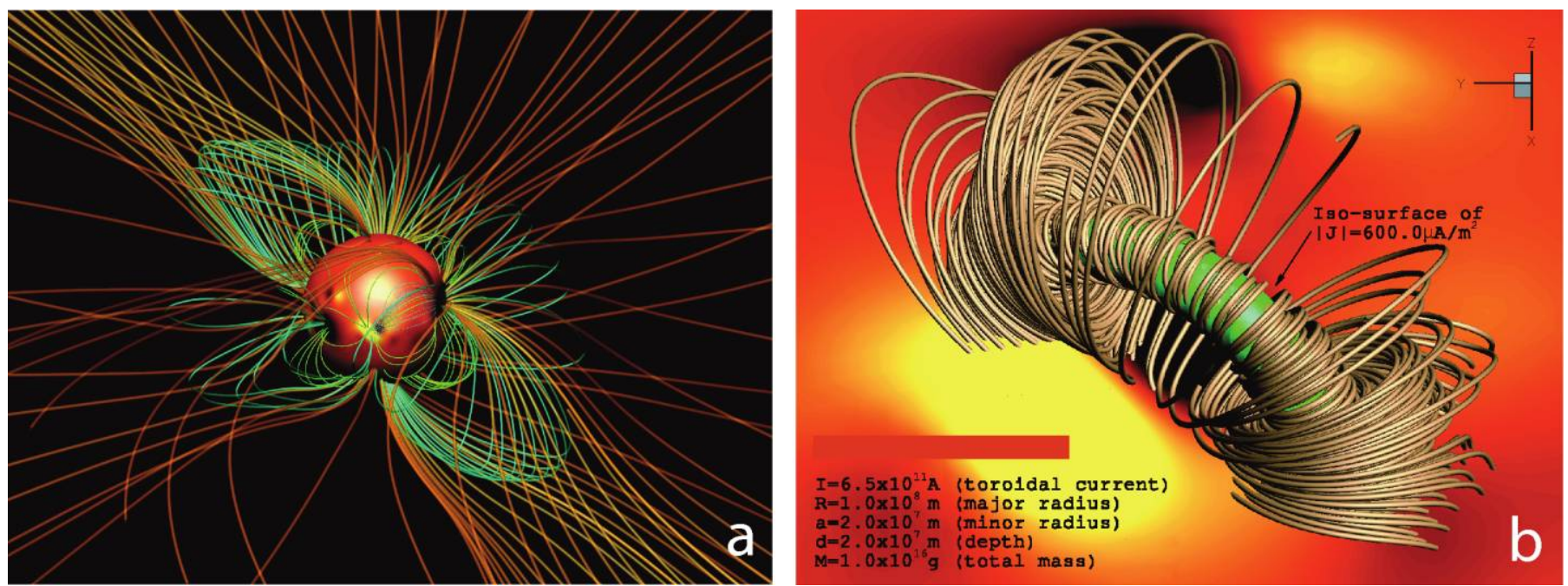

FIG. 1.- Initial condition of the corona for the October 28 CME. ( $a$ ) Radial field strength, $B_{r}$, at the base of the corona. The structure of the coronal magnetic field is illustrated with blue and yellow lines for the closed field, while orange lines show the open field. (b) Closeup of AR 10486 with the superposed flux rope illustrated with field lines and a current density isosurface colored green.

we refer the reader to this earlier paper. The simulation begins with the construction of a steady state corona and solar wind. The magnetic field at the inner boundary (base of the corona) of the SC component is specified from an MDI synoptic magnetogram centered around the time of the October 28 eruption. The initial volumetric field is specified with a potential-field-source-surface extrapolation fit to this map. The computational grid is highly refined around AR 10486, where the smallest cells are about $3 \times$ $10^{-3} R_{\odot}$. The grid is refined to $0.1 R_{\odot}$ within a $1 R_{\odot}$ diameter cylinder extending along the Sun-Earth line. The SC component is then run to steady state, allowing the solar wind to relax. Figure $1 a$ shows the steady state solution. Here the radial field strength is shown in color at the inner boundary, and field lines are drawn extending out into the corona. Orange lines represent the open field, while the closed field is shown in blue. The SC is run in the Heliographic Rotating (HGR) coordinate system with the sidereal Carrington rotation period of 25.38 days. An offset angle around the $Z$ axis places the Earth in the $-X, Z$ half-plane at the time of the large eruption on October 28.

The IH component is coupled to the $\mathrm{SC}$ component at $20 R_{\odot}$ and rapidly achieves steady state as the superfast wind blows through the domain. During the steady state run, the IH component uses a rotating coordinate system, which is then switched to Heliographic Inertial (HGI) coordinate system with an offset angle that puts the Earth in the $-X, Z$ half-plane (the orbital motion of the Earth is neglected). The grid resolution is $\frac{1}{4} R_{\odot}$ near the inner boundary and also along the Sun-Earth line within a cylinder of $1 R_{\odot}$ radius, while the largest cells are $4 R_{\odot}$.

\subsection{Generating Eruptive Events}

The October 28 event was preceded by several smaller CMEs, which significantly modified the ambient solar wind. To take into account the preconditioning effect of previous CMEs on the solar wind, we start our simulation on October 26, when a smaller CME was launched at approximately 07:00 UT from AR 10486. For this first CME, we use 07:24 UT as the initiation time, which is within the uncertainties of the observations. The much more energetic CME that is the focus of this study occurred at approximately 10:54 UT. This eruption occurred near disk center producing a full halo $\mathrm{CME}$
Here we initiate the CMEs by inserting magnetic flux ropes (the size of the active region) based on the modified Titov \& Démoulin (1999) model. In Figure $1 b$, the flux rope for the October 28 eruption is seen arching above AR 10486. The location and orientation of the flux ropes were chosen to arch over the dipolar part of the active region. The active region can be easily identified in the high-resolution synoptic map obtained from the observed photospheric magnetic field. The density in the loop was obtained from the size and estimated mass of the CMEs. The rope's magnetic field does not match that observed at the photosphere. The reason for the discrepancy is that the field of the rope is highly twisted and is orthogonal to the polarity inversion line. In contrast, the measured magnetic field was observed to be in a highly sheared configuration running parallel to the inversion line (Liu et al. 2005). In spite of this limitation, the magnetic field strength of the flux ropes can be adjusted to produce eruptions that match LASCO observations of the CME speeds at $20 R_{\odot}$ (1500 and $2500 \mathrm{~km} \mathrm{~s}^{-1}$ for the October 26 and October 28 events, respectively). For the first CME, the magnetic flux rope has a free energy of $2.3 \times 10^{32} \mathrm{ergs}$, while the second faster CME requires nearly 10 times as much energy at $2.0 \times 10^{33}$ ergs.

After 20 minutes from the start of the time-dependent simulation, the leading shock of the first CME reaches a radial distance of $5.5 R_{\odot}$ at a speed slightly exceeding $2100 \mathrm{~km} \mathrm{~s}^{-1}$. The first $\mathrm{CME}$ reaches the $\mathrm{SC} / \mathrm{IH}$ boundary at $20 R_{\odot}$ after $\sim 1.7 \mathrm{hr}$, and the speed of the leading shock is the observed $1500 \mathrm{~km} \mathrm{~s}^{-1}$. The first CME is propagating in a direction about $30^{\circ}$ off from the SunEarth line, but the flanks of the shock reach the Earth $45 \mathrm{hr}$ after the eruption at 4:30 UT Oct 28 . The simulated solar wind velocity at the Earth increases from $\sim 350$ to $550 \mathrm{~km} \mathrm{~s}^{-1}$, which is a good approximation to the solar wind conditions preceding the arrival of the October $28 \mathrm{CME}$ as observed by the $A C E$ satellite (Skoug et al. 2004; Zurbuchen et al. 2004).

The second CME is initiated at 10:54 October 28 with a flux rope that is $50 \%$ larger in radius than the previous one. Due to the larger size and stronger magnetic field, the second CME reaches a speed of $2500 \mathrm{~km} \mathrm{~s}^{-1}$ (on the Sun-Earth line) measured at $20 R_{\odot}$. After 15 minutes, the second CME reaches $9 R_{\odot}$ with a shock speed near $3200 \mathrm{~km} \mathrm{~s}^{-1}$. The shock reaches the $\mathrm{SC} / \mathrm{IH}$ boundary at $20 R_{\odot}$ in less than an hour with a speed around $2800 \mathrm{~km} \mathrm{~s}^{-1}$. 

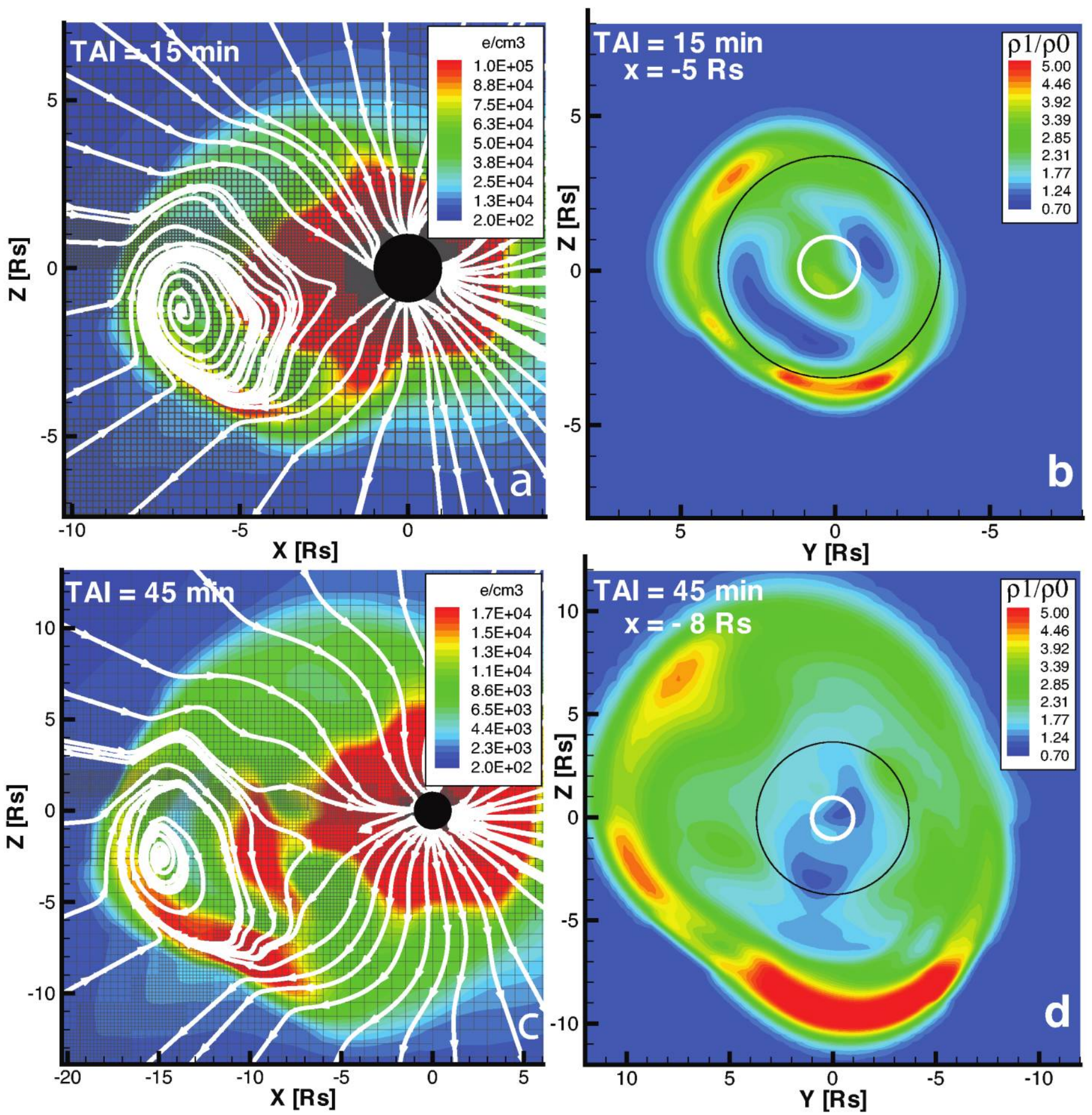

FIg. 2.- Structure of the CME at time after initiation equal to 15 and 45 minutes is shown in the top and bottom rows, respectively. $(a, c)$ Electron density is shown in color with magnetic stream lines (confined to the plane) drawn white and the numerical mesh drawn black. These images show the ejected magnetic flux rope traveling toward the Earth in the $-x$ direction with the center of the rope and densest plasma concentrations below the equatorial plane. $(b, d)$ Ratio of the density relative to the preevent state on $y-z$ planes positioned near the center of the CME at $x=-5$ and $-8 R_{\odot}$, respectively. The white and black circles are drawn at 2 and $3.7 R_{\odot}$, respectively, corresponding to the occulting disks of $\mathrm{C} 2$ and $\mathrm{C} 3$, respectively. These images show the greatest density enhancement at the bottom $(-z)$ of a nearly circular shell-type structure that evolves in a self-similar fashion.

The density structure of the October $28 \mathrm{CME}$ is shown in Figure 2. Figures $2 a$ and $2 c$ show a color representation of the electron density on the meridional plane $(x-z)$ at 15 and 45 minutes after CME initiation, respectively. White lines show the direction of the magnetic field confined to the plane. In these pictures, the magnetic flux rope is clearly seen to be expelled from the corona. Figures $2 b$ and $2 d$ show the density ratio (relative to the pre-event corona) on $y-z$ located at $x=-5$ and $-8 R_{\odot}$ at 15 and 45 minutes after initiation, respectively. The white and black circles correspond to the solar limb and LASCO C3 coronagraph, respectively. These images show the nearly self-similar evolution of the plasma expelled in the CME. The plasma is distributed in a shell with the greatest density in the southern hemisphere.

The three-dimensional density of the CME is shown in Figure 3 with isosurfaces of the density enhancement (i.e., density divided by the pre-event level) at $t=15$ minutes after initiation. Blue, 

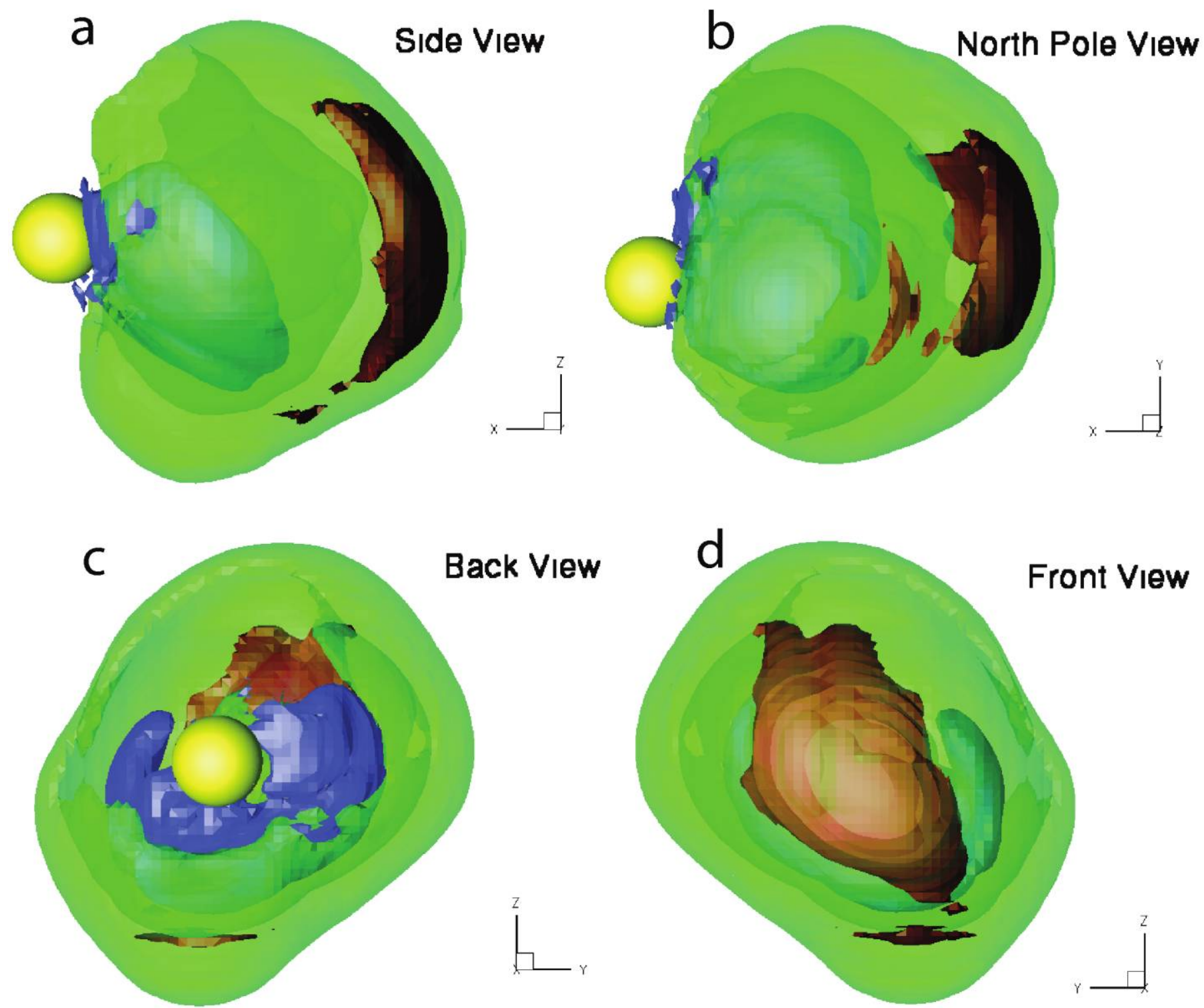

Back View

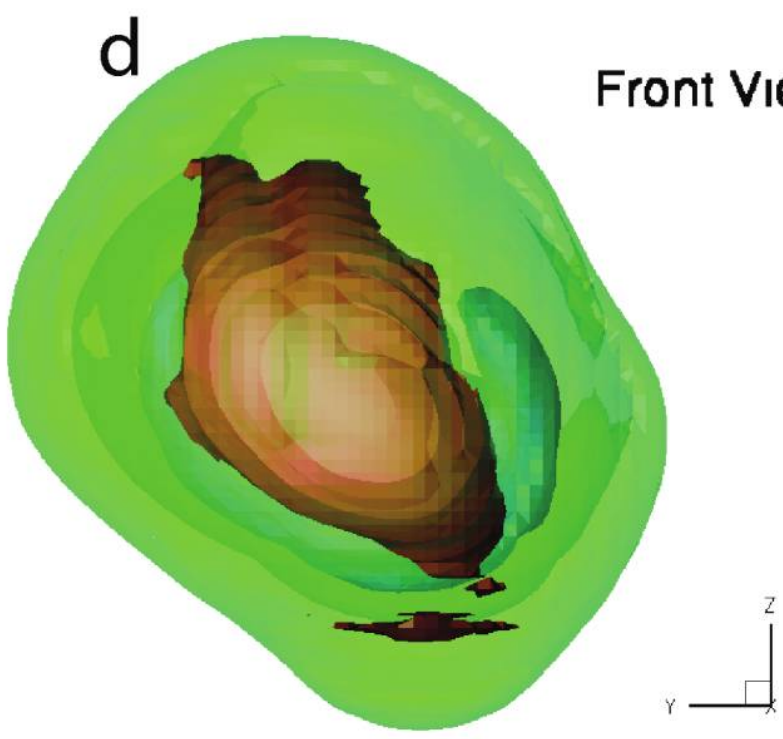

FIG. 3.- Three-dimensional density structure of the model CME 15 minutes after initiation. Isosurfaces of the density enhancement (relative to background) are shown at levels of $0.9,2$, and 5 in blue, green, and red, respectively. The green isosurface shows the extent of the shock front, while the blue isosurface shows the low-density rarefaction behind the CME. The Sun is shown with a yellow sphere.

green, and red isosurfaces corresponding to values of $0.9,2$, and 5 are shown from four perspectives: side (Fig. 3a), polar (Fig. 3b), back (Fig. 3c), and front facing Earth (Fig. 3d). The green surface effectively shows the extent of the shock front, the red surface shows the core material, while the blue isosurface shows the densitydepleted cavity. The cavity forms as a rarefaction behind the flux rope, while the densest plasma is located in the southern hemisphere, both in and below the flux rope. The polar and back views (Figs. $3 b$ and $3 c$, respectively) best show the density enhancement at the southern end of the CME. Comparison of Figure 3 with Figure 6 in Jackson et al. (2006) also suggests that the simulated $3 \mathrm{D}$ density structure qualitatively agrees with the density enhancements reconstructed from SMEI observations.

\section{COMPARISONS WITH LASCO OBSERVATIONS}

CMEs are most frequently observed in visible light that is Thomson-scattered by coronal electrons within the CME. In addition to this electron-scattered photospheric light (referred to as the $\mathrm{K}$ corona), there is also light scattered by interplanetary dust that is referred to as the $\mathrm{F}$ corona. There are significant differences between the two components of scattered light. First, the F corona is unpolarized within $5-6 R_{\odot}$, while the $\mathrm{K}$ corona is polarized with components both radial and tangential to the solar limb (Billings 1966). Second, the brightness of the $\mathrm{K}$ corona falls off much more rapidly with distance from the Sun such that it dominates close to the Sun, is roughly equal to the $\mathrm{F}$ corona at $r \approx 4 R_{\odot}$, and is much dimmer than the F corona far from the Sun. Finally, the F corona is nearly constant in time and is believed to be largely unaffected by CMEs.

The corona is optically thin in white light, so that the intensity of scattered light along a given line of sight (LOS) is the integrated contribution of all electrons along the line. The contribution to a given LOS is highly dependent on the location of the electron relative to the Sun and the observer (Billings 1966). Close to the Sun, the scattering is heavily weighted in the plane of the sky. Coronagraphs located in space observe coronal light without contending with atmospheric scattering, which makes it possible to view the corona far from the Sun. In the case of LASCO, the 


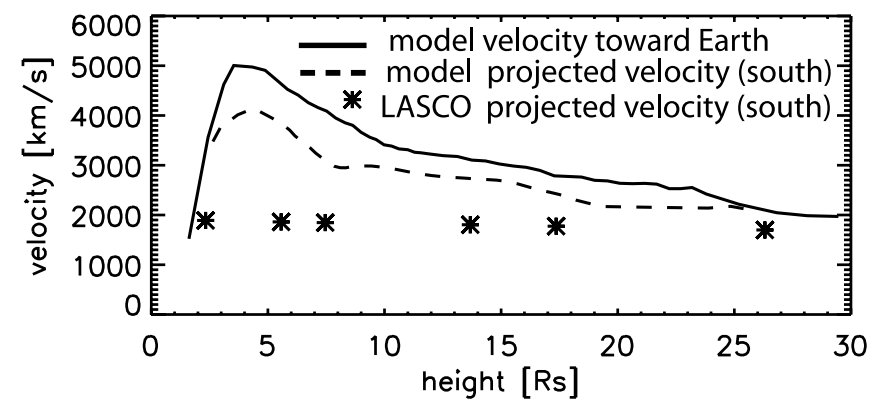

FIG. 4.-Comparison of observed and modeled CME velocity. The solid line shows the modeled CME velocity moving directly toward the Earth, while the dashed line shows the model velocity projected on the plane of the sky $177^{\circ}$ (counterclockwise) from the north polar axis. At this same location in the plane of the sky, the CME velocity is derived from LASCO observations and plotted with stars. We find that the model briefly reaches a velocity of $4000 \mathrm{~km} \mathrm{~s}^{-1}$ at $4.5 R_{\odot}$ before falling to $2000 \mathrm{~km} \mathrm{~s}^{-1}$ at $20 R_{\odot}$. In contrast, the CME is observed to decelerate from 1890 to $1699 \mathrm{~km} \mathrm{~s}^{-1}$ as it travels from 2.3 to $26.3 R_{\odot}$

$\mathrm{C} 2$ and $\mathrm{C} 3$ coronagraphs have fields of view extending to 6 and $32 R_{\odot}$, respectively. More recently, the SMEI and STEREO coronagraphs have observed Thomson-scattered light at very large angles from the Sun that extend beyond Earth's orbit.

In this section, we make comparisons between synthetic coronagraph images constructed from our model CME with LASCO observations. The synthetic images are created by numerically integrating the Thomson-scattered light along a LOS for each pixel with the appropriate scattering function (Billings 1966). Images of numerical CME models have been made in this fashion before (e.g., Wu et al. 1999; Odstrcil 2003; Manchester et al. 2004c; Lugaz et al. 2005). More recently, synthetic images have been made for models of specific CME events and qualitatively compared to LASCO images (e.g., Lugaz et al. 2007; Cohen et al. 2008). Here we go further and for the first time make truly quantitative comparisons with the data (see Fig. 4 for comparisons of CME velocity). To make the comparison, we process the synthetic data and the LASCO data in the same way and display the resulting images in identical formats. We account for $\mathrm{F}$ corona far from the Sun, where it is the dominant source of scattered light. Since our MHD model only allows us to directly treat electron density, we estimate the contribution of dust scattering with the same power-law empirical relationship used to make the LASCO images. In this case, the background F corona brightness is taken to have the form $B_{\mathrm{F}}=c r^{(0.22 \cos (2 \theta)-2.47)}$ (Koutchmy \& Lamy 1985). Here we estimate the magnitude of $c$ by setting $B_{\mathrm{F}}=B_{\mathrm{K}}$ at $r=4 R_{\odot}$, where $B_{\mathrm{K}}$ is the background polar brightness of the K corona taken from Saito et al. (1977).

The results of the comparison are shown in Figure 5. Here images of the October 28 CME as observed with LASCO C2 (Fig. 5a) and $\mathrm{C} 3$ (Figs. $5 c$ and $5 e$ ) are shown for times $t=11: 30,11: 42$, and $12: 18$, respectively. Figures $5 b, 5 d$, and $5 f$ show the synthetic images at times $t=13,21$, and 50 minutes after initiation when the model CME is closest in size to that of the corresponding LASCO image. In reality, the CME travels slower than in our model, with the observed sequence of images occurring at approximately 36 , 48, and 84 minutes after initiation. In both data and model, the color images show the total brightness divided by that of the preevent background. White circles show the solar limb, and filled black circles show the occulting disks. In all cases, we find extremely good quantitative agreement in both the magnitude and spatial distribution of the observed brightness. In these images, the greatest brightness is below the southern limb of the Sun, which extends in an arc over the eastern limb, while the dimmest part of the CME is found in the northwestern quadrant. This brightness distri- bution corresponds with the position of AR 10486, which was located at $20^{\circ}$ below the equator on October 28. By 12:18 UT, the observed brightness patterns is more highly structured than the model, showing evidence of a bright core in the south. The model (Fig. $5 f$ ) lacks these fine details and retains a single bright arc in the south.

\subsection{CME Mass}

To further quantify the comparison between the our numerical model and the LASCO coronagraph observations, we calculate the CME mass in both cases in an identical fashion. This mass is derived by integrating the excess brightness of the K corona with the assumption that the plasma is in the plane of the sky, which sets a lower limit on CME mass. The F corona is subtracted out from the observations and does not affect the mass. We restrict this integral to the brightest portion of the CME that extends from $235^{\circ}$ to $330^{\circ}$ as measured from the $-y$ axis in Figure 5. In the case of the $\mathrm{C} 2$ field of view (Fig. 5, top row), this integration sector extends from 2.0 to $6.0 R_{\odot}$, and for the $\mathrm{C} 3$ field of view (Fig. 5 , middle and bottom rows) the sector extends from 4.0 to $16.4 R_{\odot}$. With these criteria, the CME masses derived from observations at $t=11: 30,11: 42$, and 12:18 UT are $1.50 \times 10^{16}, 1.55 \times 10^{16}$, and $1.70 \times 10^{16} \mathrm{~g}$, respectively. The corresponding masses of the model CME (Figs. $5 b, 5 d$, and $5 f$ ) are, respectively, $2.23 \times 10^{16}$, $2.16 \times 10^{16}$, and $4.90 \times 10^{16} \mathrm{~g}$.

The observed masses are approximately $30 \%$ less than that of the model at times $t=11: 30$ and 11:42 UT, which is consistent with the larger filling factor of the model event. The slight decrease in model CME mass found from Figure $5 d$ is the result of a greater part of the CME being obscured by the occulting disk. By 12:18 the CME mass is observed to increase by $13 \%$, while the model mass (as shown in Fig. $5 f$ ) more than doubles. We expect the mass of fast CMEs to increase as plasma is swept up as they travel from the Sun, as shown in numerical simulations (Manchester et al. 2004c; Lugaz et al. 2005). However, the mass increase seen in LASCO observations is attributed to mass entering the coronagraph field of view from below the occulter (Vourlidas et al. 2000). In general, there is not yet proof of a plasma pileup in the low corona by CMEs. In the case of our simulation, we believe that the $\mathrm{CME}$ mass increases faster than what is observed because of the excess speed of the CME. For the observed event, the (excess) mass continues to increase by almost an order of magnitude, to $13.6 \times 10^{16} \mathrm{~g}$ as measured with SMEI on October 29, 12:00 UT, as the CME passed the Earth (Jackson et al. 2006).

\subsection{Shock Identification}

Faint arcs are frequently observed at the outer edges of fast CMEs viewed in coronagraph images, yet it has been difficult to conclusively identify these arcs as shocks. The earliest example of shock identification is that of Sime \& Hundhausen (1987), who observed a bright loop at the front of a fast CME. In that case, the presence of a shock was inferred from its high speed $\left(1070 \mathrm{~km} \mathrm{~s}^{-1}\right)$, the absence of any deflections preceding the shock, and the fact that the expanding shock front did not cease its lateral motion to form stationary legs. More recently, Vourlidas et al. (2003) observed a similar feature with LASCO that they identified as a shock wave. In this work, a numerical simulation was employed to model the shock's appearance and confirm its presence in the coronagraph image. More recent MHD simulations have also found manifestations of CME-driven shocks in synthetic coronagraph images (e.g., Manchester et al. 2004c; Lugaz et al. 2007). Perhaps the most compelling observational evidence for shocks appearing as visible components of CMEs in LASCO images is presented in Raymond et al. (2000) and Mancuso et al. (2002). Here shocks were observed 

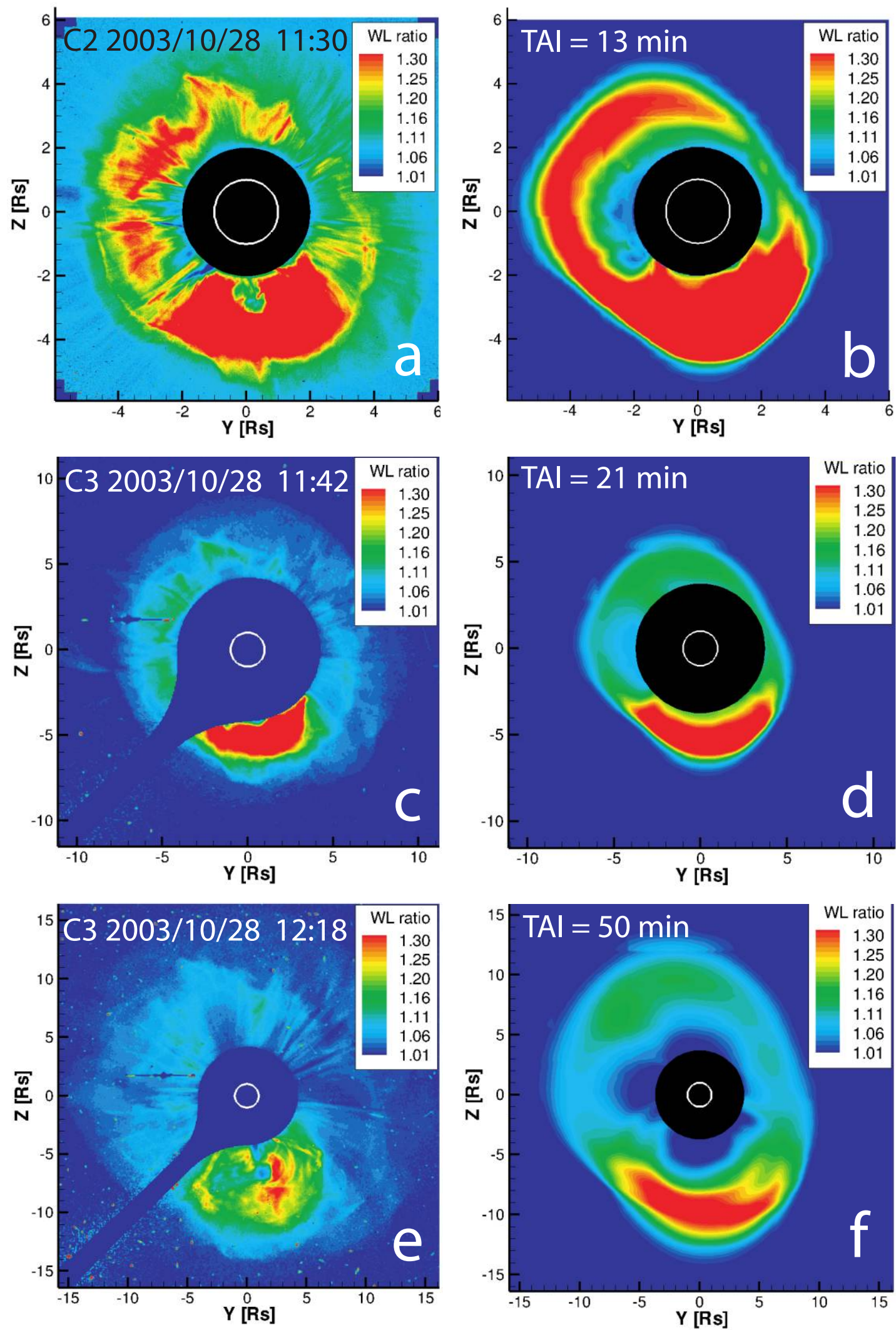

FIG. 5.-Comparison of observed (left) and simulated (right) Thomson-scattered white-light brightness. The color images show the total brightness divided by that of the pre-event background. White circles show the solar limb, and filled black circles show occulting disks. (a) LASCO C2 observation at 11:30 UT. ( $c, e$ ) LASCO C3 observations at 11:42 and 12:18, respectively. $(b, d, f)$ Model coronagraph images at 13,21, and 50 minutes after initiation. Here we find that the model achieves remarkable quantitative agreement with both the magnitude and spatial distribution of the observed brightness. 

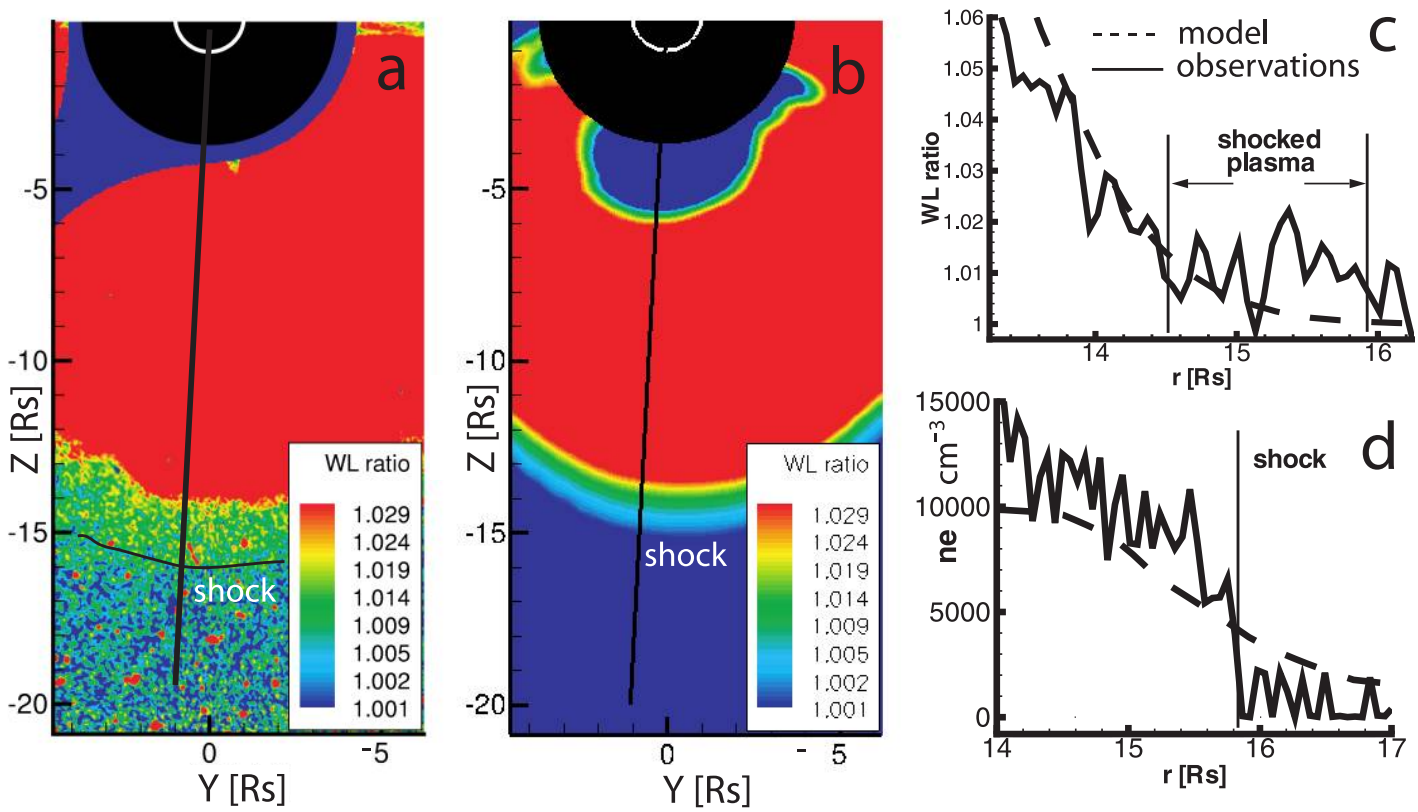

FIG. 6. - Shock structure as revealed by coronagraph images. $(a, b)$ Color images of the total brightness (divided by the pre-event brightness) for LASCO C3 (time $=$ 12:18) and for the simulation 50 minutes after initiation. The color legend is chosen to highlight the faint $(2 \%)$ increase in brightness at the shock found at the outer edge of the halo. The model shows the same faint increase in brightness at the shock as well as the capturing the width of the faint plasma sheath. $(c, d)$ Line plots of the brightness and electron density as functions of distance from Sun center along the black line at $177^{\circ}$ from the north polar axis. Model and observations are shown with dashed and solid lines, respectively. The line plots reveal that the simulation quantitatively reproduces the observed brightness increase behind the shock as well as the inferred density increase at the shock.

simultaneously in the low corona $\left(r<3 R_{\odot}\right)$ by LASCO and the Ultraviolet Coronagraph Spectrometer (UVCS), and as type II radio bursts. UVCS gave clear spectroscopic evidence for the presence of shock fronts, while radio bursts indicated the presence of shock-accelerated electrons. Recently, Pagano et al. (2008) modeled the spectral line signatures of CME-driven shocks propagating in the corona.

In our simulation, we can conclusively identify a shock front and determine its appearance in synthetic coronagraph images. By detailed comparison with the observations, we can then clearly demonstrate the existence of a shock front in the corona. For this purpose, we reexamine the coronagraph data presented in the bottom row of Figure 5. We again show the brightness, but with the color contours adjusted to more clearly show the faint features at the outermost edge of the observed and modeled CME in Figures $6 a$ and $6 b$, respectively. In both panels we see very similar faint rims at the outer edge of the CME, where the color makes a transition from blue-green and yellow, indicating an increase in brightness at a level between $1 \%$ and $2 \%$. This rim has a width of $2 R_{\odot}$ and extends out to $16 R_{\odot}$ from the Sun. In the data, this faint rim is near the noise level, and we draw a curved black line to mark its outer boundary.

For a more detailed comparison, we extract the brightness and electron density along radial lines (shown in Figs. $6 a$ and $6 b$ ) that pass through the southern outer edge of the CME. Figure $6 c$ shows both the observed and modeled brightness with solid and dashed lines, respectively. We find very good agreement between the model and the observations. Both cases exhibit a similar steep drop in brightness that levels off forming the faint rim, where the noise in the observations is close to the signal level. For the purpose of the comparison, we have aligned these features in the radial direction by shifting model results a small amount $\left(+0.62 R_{\odot}\right)$. In Figure $6 d$ we plot (solid line) the electron density deduced from observations under the assumption of an axisymmetric distribution around the Sun. The model electron density is extracted along a line extending from the center of the Sun and passing through the southernmost extent of the disturbance near $x, y, z=-8.6$, $0.83,-15.9 R_{\odot}$. The projection of this line on the $y$-z plane corresponds to the line shown in Figure $6 b$. We plot this data in Figure $6 d$ with a dashed line and translate the model density in the radial direction to align with the observed quantities. We find remarkable agreement between the two electron densities, where both increase from approximately 2000 to $10,000 \mathrm{~cm}^{-3}$ at the outer edge of the faint rim between 14 and $17 R_{\odot}$. In the simulation, we can conclusively identify this density jump as a strong fast-mode shock driven by plasma moving at $2010 \mathrm{~km} \mathrm{~s}^{-1}$. The density increase at the shock is very near the theoretical limit of $(\gamma+1) /$ $(\gamma-1)=5$ for $\gamma=1.5$ used in the simulation. The shock is smeared out over a distance of $2 R_{\odot}$ by grid resolution at $\frac{1}{4} R_{\odot}$. By a detailed comparison between the simulation and the observations, we clearly demonstrate the existence of a shock front in the LASCO C3 image at $t=12: 18$.

The overall 3D shape of the shock front is seen in Figure 3, from which we can discern why the shocked plasma is so faint. The outer edge of the shock front is $5 R_{\odot}$ from the plane of the sky and is sharply curved at its greatest distance from the $x$-axis. The lines of sight at the outer edge of the CME graze the shock surface, and even though there is nearly a factor of 5 increase in density at shock, the enhancement occurs over such a short distance that it results in a very small increase in brightness. The brightness increases as the lines of sight pass through progressively longer distances of compressed plasma behind the shock. For the numerical model, the decrease in brightness occurs faster than what is observed, in part because the shock front is smeared out by the limited numerical resolution.

\section{CME APPEARANCE AT LARGE ELONGATION: STEREO-SECCHI PREDICTIONS}

In this section, we make wide-angle, large-elongation, Thomsonscattered white-light images of the model to show how the CME 


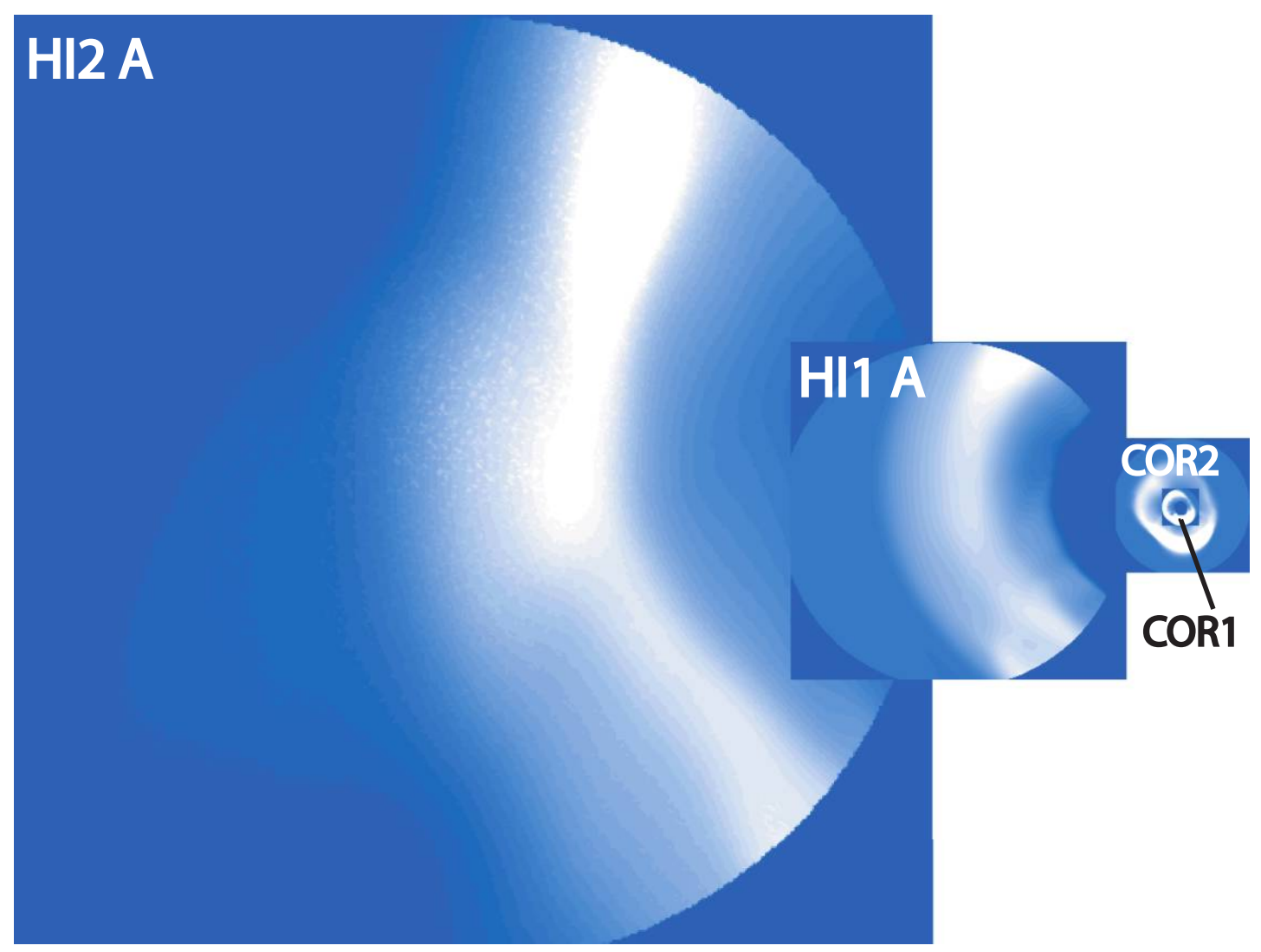

FIG. 7.- Views of the model CME as it would appear in the SECCHI coronagraphs. The CME is shown in a time series propagating through the fields of view of $\mathrm{COR} 1, \mathrm{COR} 2, \mathrm{HI} 1$, and $\mathrm{HI} 2$ that extend from 1.4 to $4 R_{\odot}, 2$ to $15 R_{\odot}, 12$ to $84 R_{\odot}\left(20^{\circ}\right.$ wide), and 66 to $381 R_{\odot}\left(70^{\circ}\right.$ wide), respectively. In this case, the $\mathrm{H}$ i images are taken from the point of view of STEREO A, $22^{\circ}$ ahead of the Earth, while COR images are made from the location of Earth.

would appear in the coronagraphs of the Sun Earth Connection Corona and Heliospheric Imager (SECCHI) on board the STEREO spacecraft (Howard et al. 2007). The fields of view of the four SECCHI coronagraphs, COR1, COR2, HI1, and HI2, give complete coverage extending from the low corona to beyond the Earth's orbit as shown in Figure 7. COR1 and COR2 coronagraphs are centered on the Sun with fields of view that extend $1.4-4$ and $2-15 R_{\odot}$, respectively. HI1 and HI2 are pointed toward the Sun-to-Earth line with fields of view that extend $12-84$ and 66-381 $R_{\odot}$, respectively. Figure 7 shows synthetic COR1 and COR2 images of our model of the October $28 \mathrm{CME}$ as it appears from the location of the Earth. HI1 and HI2 images show the CME from a location $22^{\circ}$ ahead of the Earth, where STEREO A will be one year into its mission.

Thomson scattering has a strong angular dependence such that scattering into a LOS is highly dependent on the location of the electron relative to the Sun and the observer (Billings 1966). The region of space for which there is maximum scattering into the LOS is in the shape of a sphere that contains the Sun and the observer as seen in Figure 10. Close to the Sun, the scattering is heavily weighted to the plane of the sky. At large elongation (more than $60 R_{\odot}$ from the Sun), the spherical shape of the maximum scattering surface becomes significant to the appearance of coronagraph images (Vourlidas \& Howard 2006).

Our model provides an opportunity to examine the effects of the angular dependence of Thomson scattering on the appearance of CMEs at large elongation as seen in the HI2 coronagraphs. Figure 8 shows the time evolution of the model CME as it would appear from SECCHI-HI2A one year into the mission $\left(22^{\circ}\right.$ ahead of the Earth). The total brightness is shown in a time sequence at intervals $t=9.1,13.1,18.3$, and $22.3 \mathrm{hr}$ in Figures $8 a-8 d$, respectively. The CME is seen to propagate into the field of view on the right-hand side of Figure $8 a$, appears to stall in Figure $8 b$, and then reappears on the left-hand sides of Figures $8 c$ and $8 d$ without ever being bright in the middle of the field of view. (See the electronic edition of the Journal for an mpeg animation of this figure.) Figure 9 shows the corresponding series of images that would be made by SECCHI-HI2B. In this case, the spacecraft is $22^{\circ}$ behind the Earth with the HI2B instrument pointing toward the Sun-Earth line in the opposite direction as HI2A. From this perspective, the CME enters the HI2B field of view from the lefthand side seen in Figure $9 a$. Here the CME appears as an arc that first brightens, as seen in Figure $9 b$, and then gradually fades away, as seen in Figures $9 c$ and $9 d$.

The complex behavior shown in the model HI2 images is not seen in standard coronagraph images and was not found in the simulated HI2 images of a less structured CME examined by Lugaz et al. (2005). The reason the CME fades in and out is because of multiple crossings of the Thomson sphere by dense arcs that propagate through the field of view of HI2A as illustrated in Figure 10 (Vourlidas \& Howard 2006). Here, for the same time sequence as shown in Figure 8, we show the mass density in color on the equatorial $(x-y)$ plane. We mark the intersection of the Thomson sphere with the equatorial plane with a white circle. The position of STEREOA is marked along with black lines roughly showing the field of view of the HI2 coronagraph. Dense plasma is preferentially brightened in close proximity to the Thomson sphere. At $t=9.1 \mathrm{hr}$, a dense arc of plasma crosses the Thomson sphere from the right-hand side. This arc begins to move away from the Thomson sphere followed by a cavity at times $t=13.1$ and $18.3 \mathrm{hr}$, 

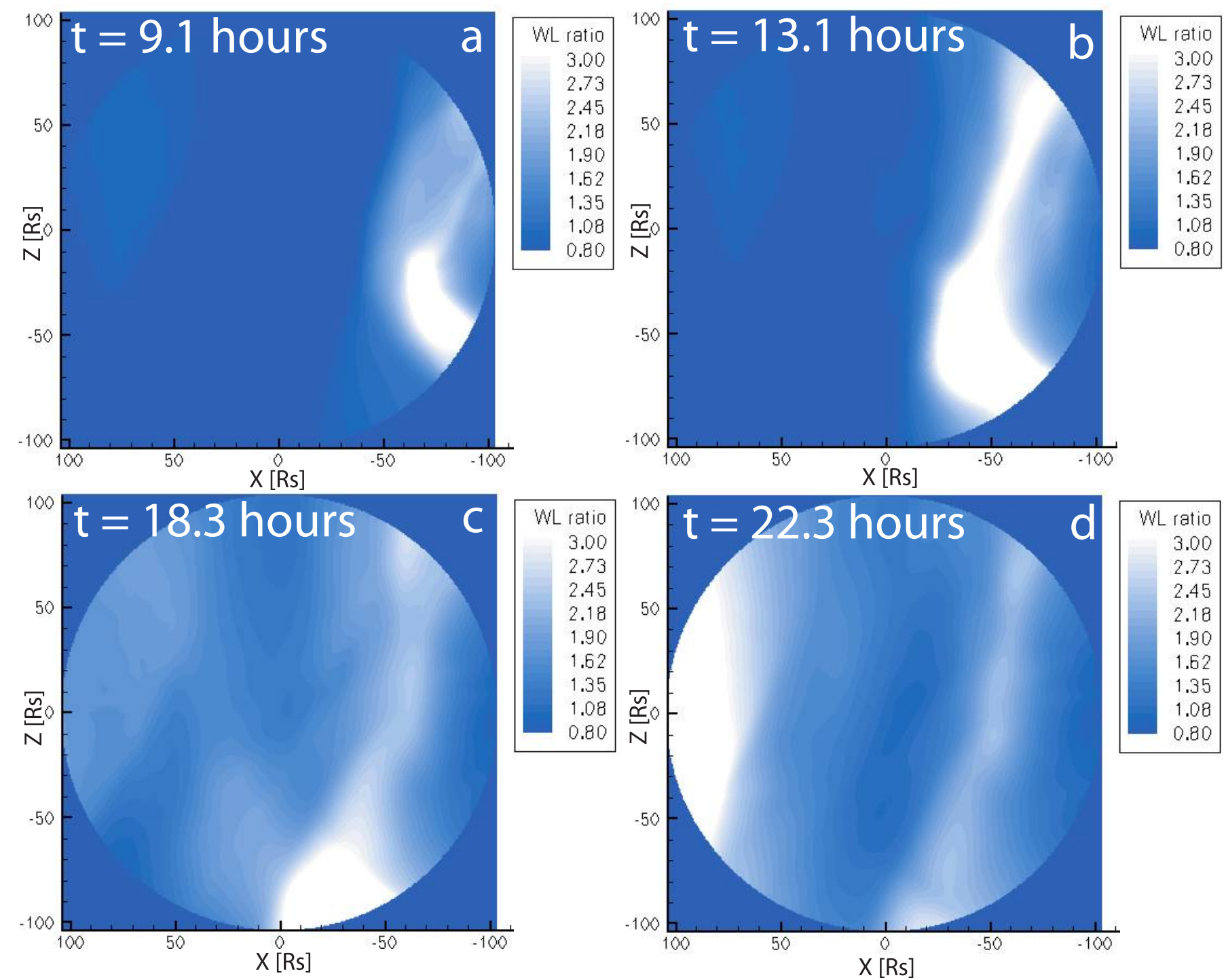

FIG. 8.- Synthetic image of the CME as it would appear from SECCHI-HI2A one year into the mission ( $22^{\circ}$ ahead of the Earth). The total brightness is shown in a time

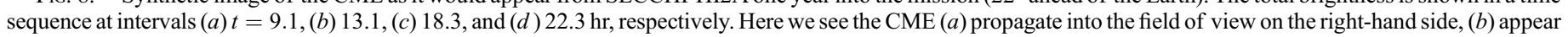

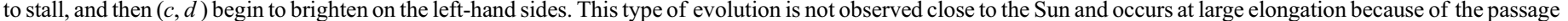

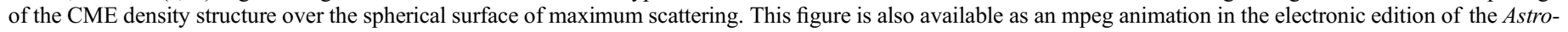
physical Journal. 

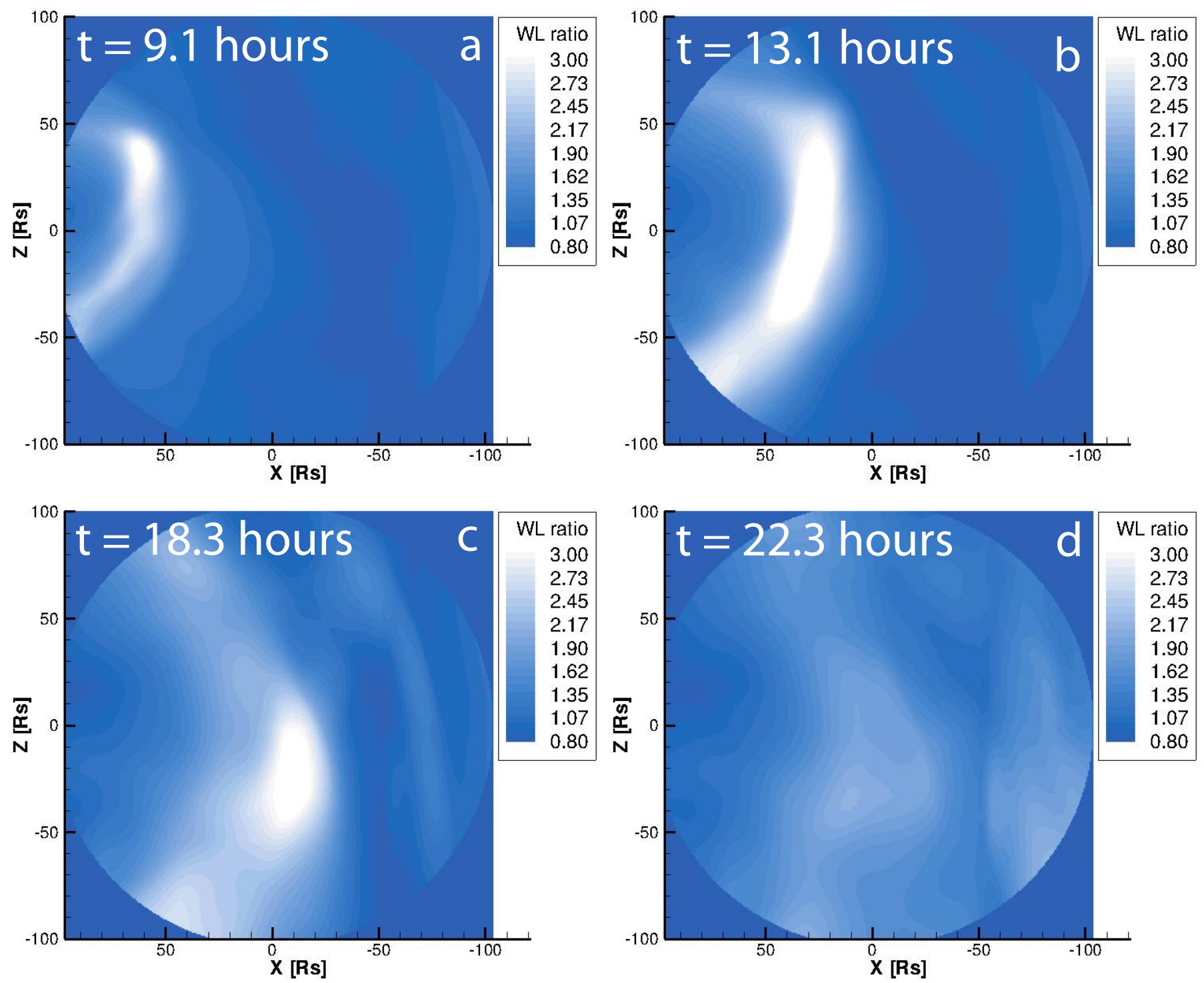

FIG. 9.- Synthetic image of the CME as it would appear from SECCHI-HI2B one year into the mission $\left(22^{\circ}\right.$ behind the Earth). The total brightness is shown in a time sequence at intervals $(a) t=9.1,(b) 13.1,(c) 18.3$, and $(d) 22.3 \mathrm{hr}$, respectively. Here we see the CME (a) propagate into the field of view on the left-hand side, (b) noticably brighten, $(c)$ fade, and then $(d)$ all but disappear. This evolution is much different than that shown in Fig. 8 and illustrates the 3D structure viewed in the opposite direction as seen by the HI2A coronagraph. 

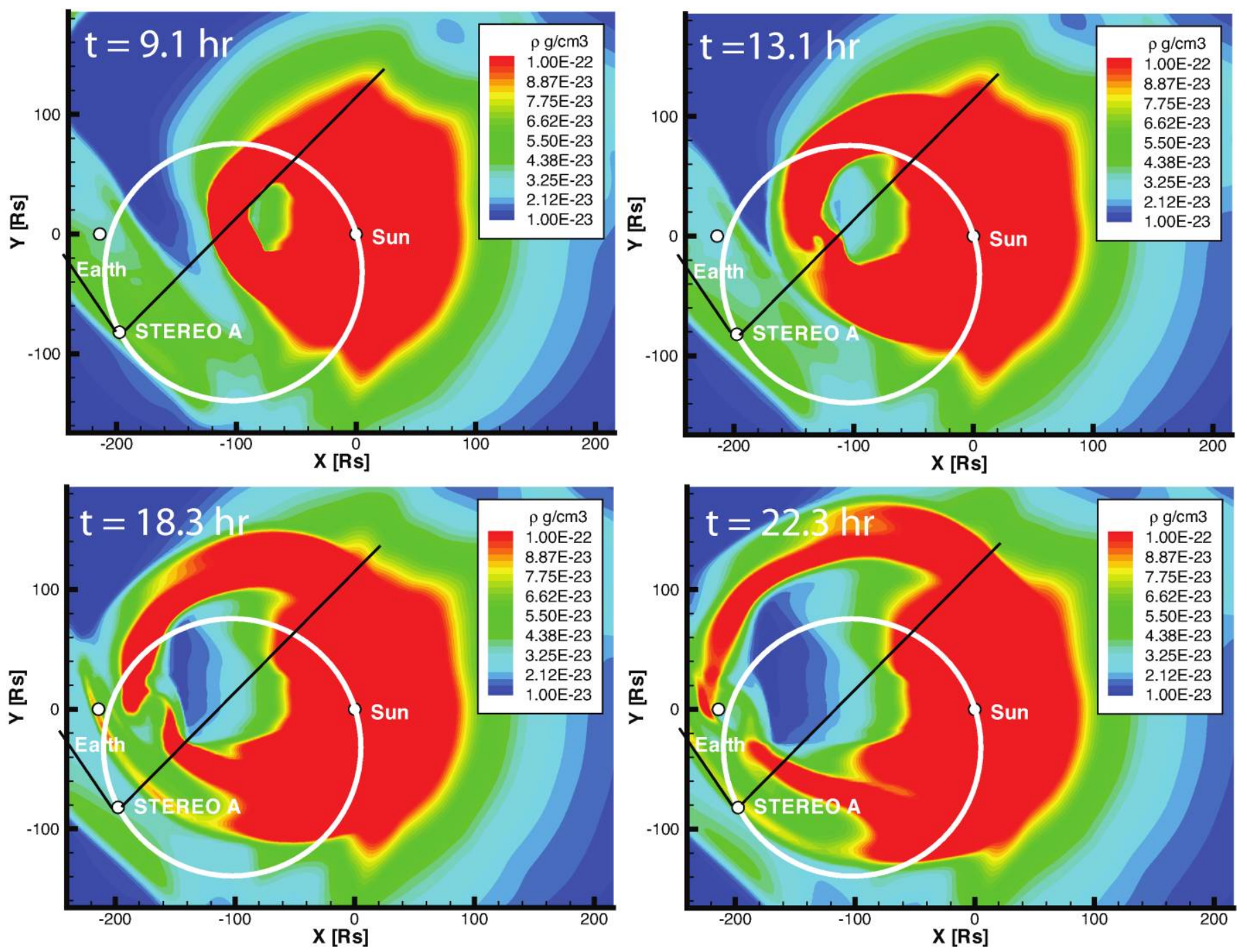

FIG. 10.- Time evolution of the density structure of the CME on the equatorial plane. The density is shown in color in a time sequence at intervals $(a) t=9.1,(b) 13.1$, (c) 18.3 , and $(d) 22.3 \mathrm{hr}$, respectively. The locations of the Sun, Earth, and STEREO A and its field of view are shown along with the equator of the Thomson sphere, the location of maximum scattering into the observer's LOS. The panels correspond to the same times shown in Fig. 8 and clearly show how dense plasma crosses the Thomson sphere to produce the time evolution of brightness seen in Fig. 8.

which explains the dimming on the right-hand side of the field of view. By $t=22.3 \mathrm{hr}$, dense plasma on the Thomson sphere is found near the spacecraft on the left-hand side and far across the Thomson sphere on the right-hand side. This two-sided distribution of plasma and its proximity to the Thomson sphere coincides with the bright arcs seen in the left- and right-hand sides of the field of view seen in Figure $8 d$.

\section{SUMMARY AND CONCLUSIONS}

We have preformed a detailed analysis of a 3D MHD timedependent simulation of flux-rope-driven CMEs originating from AR 10408 on 2003 October 26 and 28 (Tóth et al. 2007). The simulation was carried out with the SWMF in which propagation of the CMEs were followed from the low corona to beyond $1 \mathrm{AU}$. We have created synthetic coronagraph images of the October 28 event and made strict quantitative comparisons of these images with those made my LASCO C2 and C3. The simulation closely reproduces the appearance of the October $28 \mathrm{CME}$, in both the morphology and magnitude of Thomson-scattered light through the fields of view of both LASCO C2 and C3. Furthermore, the CME masses calculated from the synthetic coronagraph images and the LASCO observations also are in close agreement.
We have identified a shock front at the southern edge of the CME. A detailed analysis shows that the appearance of the CMEdriven shock in a synthetic coronagraph image closely matches the same structure found in the LASCO C3 image at 12:18 UT on October 28. Both the rigor of this quantitative comparison and the fidelity in reproducing coronagraph images of CMEs is unprecedented. This is all the more remarkable in that there was no parameter fitting to produce the $\mathrm{CME}$ images, as only the $\mathrm{CME}$ speed at $20 R_{\odot}$ was used as a guide in specifying the flux rope.

The simulation allows the opportunity to view the October 28 CME as it would appear at large elongation in the HI2 coronagraph (Lugaz et al. 2005). Here we found complex time evolution of the CME in the HI2 field of view, as dense structures were temporarily highlighted as they came in close proximity to the Thomson sphere. These results vividly illustrate the effects of CME propagation viewed at large elongation pointed out by Vourlidas \& Howard (2006).

An aspect of the simulation that can be improved on is the speed of the CME, which is a factor of 2 too fast inside of $5 R_{\odot}$, and does not recover the measured velocity until $20 R_{\odot}$. We conclude that most of the errors in CME speed originate from the ad hoc initiation with a flux rope in a state of force imbalance. However, 
the expansion of the flux rope, in the realistic 3D coronal model, is remarkable in its ability to reproduce the appearance of the $\mathrm{CME}$ in Thomson-scattered white light. This result suggests that much of the density structure of the CME is determined by the plasma distribution in the corona surrounding the active region.

Future models of CME initiation will be improved by the use vector magnetogram data rather than just fitting flux ropes to the observed line-of-sight component of the field. For this event, vector magnetograms clearly show that the magnetic field of AR 10486 is very highly sheared, with the field running nearly parallel to the neutral line from which the CMEs erupt (Liu \& Hayashi 2006). The evolution of magnetic shear is made manifest by observations of photospheric proper motions, which show strong shear flows along the magnetic neutral line prior to CMEs (Yang et al. 2004; Deng et al. 2006). These observations strongly suggest that the magnetic shear is ultimately driving these CMEs. A physical explanation and simulation of such shear flows was first provided, respectively, by Manchester \& Low (2000) and Manchester (2001), which shows that the flows are driven by the Lorentz force that occurs as the magnetic field emerges in a stratified atmosphere.
Furthermore, these Lorentz-force-driven flows may persist until the magnetic field becomes so highly sheared that there is a loss of equilibrium, producing eruptions that can drive CMEs (Manchester 2003; Manchester et al. 2004a). The need to resolve the photospheric pressure scale height has limited these simulations to Cartesian domains. Currently, we are addressing the challenge to include this self-consistent shearing mechanism in a global model of CME initiation that is guided by vector magnetic field observations.

This work has been supported in part by NASA AISRP grant NNG04GP89G, NASA STEREO grant NAS5-3131, NSF ITR project ATM-0325332, and by DoD MURI grant F49620-01-10359. W. M. and M. O. are thankful for support provided by LWS grant NNX06AC36G. G. T. has been partially supported by the Hungarian Science Foundation (OTKA grant T047042). We acknowledge the use of data from the $S O H O \mathrm{C} 2$ and $\mathrm{C} 3$ coronagraphs. We gratefully acknowledge the supercomputing resources provided by NASA's Columbia system under award SMD1-Dec04-0099 (OTKA grant T047042).
Billings, D. W. 1966, A Guide to the Solar Corona (San Diego: Academic Press)

Cohen, O., Sokolov, I. V., Roussev, I. I., Lugaz, N., Manchester, W. B., Gombosi, T. I., \& Arge, C. N. 2008, J. Atmos. Sol.-Terr. Phys., 70, 583

Cohen, O., et al. 2007, ApJ, 654, L163

Deng, N., Xu, Y., Yang, G., Cao, W., Liu, C., Rimmele, R., Wang, H., \& Denker, C. 2006, ApJ, 644, 1278

Dryer, M., Smith, Z., Fry, C. D., Sun, W., Deehr, C. S., \& Akasofu, S.-I. 2004 Space Weather, 2, S09001, DOI: 10.1029/2004SW000087

Gombosi, T. I., Tóth, G., De Zeeuw, D. L., Hansen, K., Kabin, K., \& Powell, K. G. 2001, J. de Chim. Phys., 177, 176

Groth, C. P. T., De Zeeuw, D. L., Gombosi, T. I., \& Powell, K. G. 2000, J. Geophys. Res., 105, 25053

Howard, R. A., et al. 2007, Space Sci. Rev., 105, 25053

Intriligator, D. S., Sun, W., Dryer, M., Fry, C. D., Deehr, C., \& Intriligator, J. 2005, J. Geophys. Res., 110, A09S10, DOI: 10.1029/2004JA010939

Jackson, B. V., Buffington, A., Hick, P. P., Wang, X., \& Webb, D. 2006, J. Geophys. Res., 111, A04S91, DOI: 10.1029/2004JA010942

Jacobs, C., van der Holst, B., \& Poedts, S. 2007, A\&A, 470, 359, DOI: 10.1051/0004-6361:20077305

Koutchmy, S., \& Lamy, P. L. 1985, in Properties and Interactions of Interplanetary Dust, ed. R. H. Giese \& P. L. Lamy (Dordrecht: Reidel), 63

Krall, J., Yurchyshyn, V. B., Slinker, S., Skoug, R. M., \& Chen, J. 2006, ApJ, 642,541

Liu, C., Deng, N., Liu, Y., Falconer, D., Goode, P., Denker, C., \& Wang, H. 2005, ApJ, 622, 722

Liu, Y., \& Hayashi, K. 2006, ApJ, 640, 1135

Lugaz, L., Manchester, W. B., IV, \& Gombosi, T. 2005, ApJ, 627, 1019

Lugaz, L., Manchester, W. B., IV, Roussev, I. I., Tóth, G., \& Gombosi, T. 2007, ApJ, 659, 788

Luhmann, J. G., Solomon, S. C., Linker, J. A., Lyon, J. G., Mikic, Z., Odstrcil, D., Wang, W., \& Wiltberger, M. 2004, J. Atmos. Terr. Phys., 66, 1243

Manchester, W., IV. 2001, ApJ, 547, 503

. 2003, J. Geophys. Res., 108, A01162, DOI: 10.1029/2002JA009252

Manchester, W., IV, Gombosi, T., DeZeeuw, D., \& Fan, Y. 2004a, ApJ, 610, 588

Manchester, W., IV, Gombosi, T. I., Ridley, A. J., Roussev, I., DeZeeuw, D. L., Sokolov, I. V., Powell, K. G., \& Tóth, G. 2004b, J. Geophys. Res., 109, A02107, DOI: 10.1029/2003JA010150

Manchester, W., IV, Gombosi, T. I., Roussev, I., DeZeeuw, D. L., Sokolov, I. V., Powell, K. G., Tóth, G., \& Opher, M. 2004c, J. Geophys. Res., 109, A01102, DOI: $10.1029 / 2002 J A 009672$

Manchester, W., IV, \& Low, B. C. 2000, Phys. Plasmas, 7, 1263

Mancuso, S., Raymond, J. C., Kohl, J., Ko, Y.-K., Uzzo, M., \& Wu, R. 2002, A\&A, 383, 267
Odstrcil, D. 2003, Adv. Space Res., 32, 497

Odstrcil, D., Pizzo, V. J., \& Arge, C. N. 2005, J. Geophys. Res., 110, A02106, DOI: $10.1029 / 2004 J A 010745$

Pagano, P., Raymond, J. C., Reale, F., \& Orlando, S. 2008, A\&A, 481, 835

Powell, K. G., Roe, P. L., Linde, T. J., Gombosi, T. I., \& De Zeeuw, D. L. 1999, J. de Chim. Phys., 154, 284

Raymond, J. C., et al. 2000, Geophys. Res. Lett., 1439

Riley, P., Linker, J. A., Mikić, Z., Odstrcil, D., Pizzo, V. J., \& Webb, D. F. 2002, ApJ, 578, 972

Roussev, I. I., Forbes, T. G., Gombosi, T. I. Sokolov, I. V., De Zeeuw, D. L., \& Birn, J. 2003a, ApJ, 588, L45

Roussev, I. I., Gombosi, T. I. Sokolov, I. V., Velli, M., Manchester, W., De Zeeuw, D. L., Liewer, P., \& Tóth, G. 2003b, ApJ, 595, L57

Saito, K., Poland, A. I., \& Munro, R. 1977, Sol. Phys., 55, 121

Sime, D. G., \& Hundhausen, A. J. 1987, J. Geophys. Res., 92, 1049

Skoug, R. M., Gosling, J. T., Steinberg, J. T., McComas, D. J., Smith, C. W., Ness, N. F., Hu, Q., \& Burlaga, L. F. 2004, J. Geophys. Res., 109, A09102 Su, Y. N., Golub, L., Van Ballegooijen, A. A., \& Gros, M. 2006, Sol. Phys., 236,325

Titov, V. S., \& Démoulin, P. 1999, A\&A, 351, 707

Tokumaru, M., Kojima, M., Fujiki, K., Yamashita, M., \& Jackson, B. 2007, J. Geophys. Res., 112, A05106, DOI: 10.1029/2006JA012043

Toth, G., De Zeeuw, D., Gombosi, T., Manchester, W., IV, Ridely, A., Sokolov, I., \& Roussev, I. 2007, Space Weather, 5, S06003

Toth, G., De Zeeuw, D. L., Gombosi, T. I., \& Powell, K. 2006, J. de Chim. Phys., 217, 722

Toth, G., et al. 2005, J. Geophys. Res., 110, A12226, DOI: 10.1029/2005JA011126 Usmanov, A. V., \& Dryer, M. 1995, Sol Phys., 159, 347

Usmanov, A. V., Goldstein, M. L., Besser, B. P., \& Fritzer, J. M. 2000, J. Geophys. Res., 105, 12675

Vourlidas, A., \& Howard, R. A. 2006, ApJ, 642, 1216

Vourlidas, A., Subramanian, Dere, K. P., \& Howard, R. A. 2000, ApJ, 534, 456

Vourlidas, A., Wu, S. T., Wang, A. H., Subramanian, P., \& Howard, R. A. 2003, ApJ, 598, 1392

Wang, Y.-M., \& Sheeley, N. R. 1990, ApJ, 355, 726

Wu, C.-C., et al. 2005, J. Geophys. Res., 110, A09S17, DOI: 10.1029/2005JA011011

Wu, S. T., Guo, W. P., Michels, D. J., \& Burlaga, L. F. 1999, J. Geophys. Res., 14789,1999

Yang, G., Xu, Y., Cao, W., Wang, H., Denker, C., \& Rimmele, T. R. 2004, ApJ, 617, L151

Zurbuchen, T. H., Gloeckler, G., Ipavich, F., Raines, J., Smith, C. W., \& Fisk, L. A. 2004, Geophys. Res. Lett., 31, L11805, DOI: 10.1029/2004GL019461 